\title{
Effect of New Herbicides and Herbicide Mixtures on Growth and Yield of Transplanted Rice
}

\author{
B. Venkatesh*, Y. S. Parameswari, M. Madhavi and T. Ram Prakash \\ Department of Agronomy, College of Agriculture, Rajendranagar, Hyderabad-500030, \\ Professor Jayashankar Telangana State Agricultural University, India \\ *Corresponding author
}

\section{A B S T R A C T}

\begin{tabular}{l} 
K e y w o r d s \\
Herbicides, \\
Transplanted rice, \\
Growth parameters, \\
Yield attributes and \\
Yield \\
\hline Article Info \\
$\begin{array}{l}\text { Accepted: } \\
\text { 20 June } 2020 \\
\text { Available Online: } \\
\text { 10 July } 2020\end{array}$ \\
\hline
\end{tabular}

\section{Introduction}

Rice (Oryza sativa L.) is the most important staple food for more than half of the world population. The warm and humid climatic conditions are prevailing in most part of Asia are well suited to rice production. India is the world second largest producer $(105.3 \mathrm{M} \mathrm{t})$ covering an area of $43.10 \mathrm{~m}$ ha with productivity level of $2.38 \mathrm{tha}^{-1}$. However, the productivity of rice in India is very low compared to other rice growing countries like
China (7.3 t ha $\left.{ }^{-1}\right)$, Australia $\left(10.1 \mathrm{t} \mathrm{ha}^{-1}\right)$, U.S $\left(7.5 \mathrm{t} \mathrm{ha}^{-1}\right)$ and Russia $\left(5.2 \mathrm{t} \mathrm{ha}^{-1}\right.$ ) (Yadav et al., 2019). Rice is cultivated in different ecosystems to increase production levels due to climate change. Though different ecosystems are emerging day by day but, transplanting is the most dominant and traditional method of rice cultivation under irrigation. Weeds are the major constraints in rice production. Transplanted rice is infested by heterogeneous type of weed flora which causes yield reduction about 33-45 percent 
(Duray et al., 2015). Hand weeding though efficient, it is expensive, time consuming, difficult task and often limited by scarcity of labour at critical period may lead to unsatisfactory weed control. To get rid this hurdle usage of herbicides offer a selective and economical control of weeds right from beginning of crop.

Due to continuous usage of same mode of action of herbicides like butachlor, pretilachlor etc. weed shift is observed in transplanted rice. Weed shift from grasses to broad-leaf weeds and sedges were observed in transplanted rice due to continuous use of same herbicides (Mohapatra et al., 2017). All traditional herbicides are narrow spectrum of control and at the same time dosage of herbicides is more compare to new low dose herbicides and pre-mix herbicides and also cause herbicide residues in soil leads to environmental pollution. Keeping in this view the present experiment was conducted to study the effect of new herbicides and herbicide mixtures on growth and yield of transplanted rice.

\section{Materials and Methods}

A field experiment was conducted at College Farm, College of Agriculture Rajendranagar, Professor Jayashankar Telangana State Agricultural University, Rajendranagar, Hyderabad during Kharif-2019. The farm is geographically situated at $17^{0} 19^{\prime} 16.4$ " North latitude and $78^{\circ} 24^{\prime} 43^{\prime \prime}$ East longitudes and at an altitude of $542.3 \mathrm{~m}$ above mean sea level. According to troll's climatic classification, it falls under semi- arid tropics (SAT). The soil of experimental site was sandy loam in texture with $\mathrm{p}^{\mathrm{H}}$ of 7.85 , low available nitrogen (235.2), medium phosphorus (38.8) and high potassium content (379). The experiment was consisted of twelve weed management practices laid out in randomized block design with three replications. RNR -
15048 (Telangana sona) variety was transplanted in main field on 8 th August at the age of 28 days old seedlings with a spacing of 15 X $10 \mathrm{~cm}$. All pre-emergence herbicides were applied within three days after transplanting and post emergence herbicides treatments were applied at $2-3$ leaf stage of weeds. During crop growing period (July $10^{\text {th }}$ to November $17^{\text {th }}$ ) a total rainfall of $693.5 \mathrm{~mm}$ received in 45 rainy days. The data on growth parameters and yield was recorded randomly selected five plants from net plot. The data was statistically analysed.

\section{Results and Discussion}

\section{Growth parameters}

The data related to growth parameters were significantly influenced by different weed management practices over un weeded control. The higher plant height, number of tillers and dry matter production were registered with hand weeding at 20 and 40 DAT which was statistically on par with flopyrauxifen- benzyl + cyhalofop butyl $10 \%$ EC $150 \mathrm{~g} \mathrm{ha}^{-1}$ (PoE) $f b$ hand weeding at 40 DAT, penoxsulam $1.02 \%\left(20 \mathrm{~g} \mathrm{ha}^{-1}\right)+$ cyhalofop butyl 5.1\% OD (100 $\left.\mathrm{g} \mathrm{ha}^{-1}\right)$ (PoE) $f b$ hand weeding at 40 DAT and flopyrauxifen- benzyl + penoxsulam $12 \%$ EC $40.64 \mathrm{~g} \mathrm{ha}^{-1}$ (PoE) $f b$ hand weeding at 40 DAT. And these treatments superior over remaining treatments.

These treatments followed by pyrazosulfuronethyl $0.15 \% @ 15 \mathrm{~g} \mathrm{ha}^{-1}+$ pretilachlor $6 \%$ GR @ $600 \mathrm{~g} \mathrm{ha}^{-1}$ (PE) $f b$ hand weeding at 30 DAT, orthosulfamuron + pretilachlor $6 \%$ @ $600 \mathrm{~g}^{-1} \mathrm{ha}$ (PE) $f b$ hand weeding at 30 DAT and penoxsulam $0.97 \%$ @ $20 \mathrm{~g} \mathrm{ha}^{-1}+$ butachlor $38.8 \%$ SE @ $820 \mathrm{~g} \mathrm{ha}^{-1}$ (PE) $f b$ hand weeding at 30 DAT and they were on par with each other. Then followed by ipfencarbazone $25 \%$ SC @ $156.25 \mathrm{~g} \mathrm{ha}^{-1}$ (PE) $f b$ hand weeding at $30 \mathrm{DAT}$, penoxsulam 
$2.65 \%$ OD @ $25 \mathrm{~g} \mathrm{ha}^{-1}$ (PoE) $f b$ hand weeding at 40 DAT, bispyribac-sodium $10 \%$ SC $25 \mathrm{~g} \mathrm{ha}^{-1}$ (PoE) $f b$ hand weeding at 40 DAT and pretilachlor (PE) $50 \%$ EC @ 0.75 $\mathrm{kg}$ ha ${ }^{-1} f b 2,4-\mathrm{D} 1.0 \mathrm{~kg} \mathrm{ha}^{-1}$ (PoE). Un weeded control plot recorded significantly lower plant height, number of tillers and dry matter production. Among the weed management practices application of herbicide mixture $f b$ hand weeding recorded higher growth parameters compared to single herbicides $f b$ hand weeding. This might be due to control of complex weed flora in time and avoids competition so, resulted in higher tillers and crop dry matter production. These results were in line with Yakadri et al., (2016) and Rana et al., (2018).

Table.1 Effect of new herbicides and herbicide mixtures on growth parameters of transplanted rice

\begin{tabular}{|c|c|c|c|}
\hline Treatments & $\begin{array}{c}\text { Plant } \\
\text { height } \\
(\mathrm{cm})\end{array}$ & $\begin{array}{c}\text { No. of } \\
\text { tillers (No. } \\
\left.\text { m }^{-2}\right)\end{array}$ & $\begin{array}{l}\text { Dry matter } \\
\left(\mathrm{Kg} \mathrm{ha}^{-1}\right)\end{array}$ \\
\hline $\begin{array}{l}\mathrm{T}_{1} \text { - Penoxsulam } 0.97 \%\left(20 \mathrm{~g} \mathrm{ha}^{-1}\right)+\text { butachlor }(38.8 \%) \text { SE } 820 \mathrm{~g} \mathrm{ha}^{-1}(\mathrm{PE}) \mathrm{fb} \\
\text { HW at } 30 \text { DAT }\end{array}$ & 94.7 & 354 & 13375 \\
\hline $\begin{array}{l}\mathrm{T}_{2} \text { - Pyrazosulfuron-ethyl } 0.15 \%\left(15 \mathrm{~g} \mathrm{ha}^{-1}\right)+\text { pretilachlor } 6 \% \text { GR (600g ha- } \\
\text { 1) (PE) } f b \text { HW at } 30 \text { DAT }\end{array}$ & 97.0 & 370 & 13517 \\
\hline $\begin{array}{l}\text { T }_{3} \text { - Orthosulfamuron + pretilachlor } 6 \%\left(600 \mathrm{~g} \mathrm{ha}^{-1}\right) \mathrm{GR}(\mathrm{PE}) \mathrm{fb} \mathrm{HW} \text { at } 30 \\
\text { DAT }\end{array}$ & 95.8 & 364 & 13453 \\
\hline $\mathrm{T}_{4}$ - Ipfencarbazone $25 \%$ SC $156.25 \mathrm{~g} \mathrm{ha}^{-1}(\mathrm{PE}) \mathrm{fb} \mathrm{HW}$ at $30 \mathrm{DAT}$ & 89.7 & 338 & 12568 \\
\hline $\mathrm{T}_{5}$ - Penoxsulam $2.65 \%$ OD $25 \mathrm{~g} \mathrm{ha}^{-1}(\mathrm{PoE}) f b \mathrm{HW}$ at $40 \mathrm{DAT}$ & 89.0 & 330 & 12474 \\
\hline $\begin{array}{l}\mathrm{T}_{6} \text { - Penoxsulam } 1.02 \%\left(20 \mathrm{~g} \mathrm{ha}^{-1}\right)+\text { cyhalofop butyl } 5.1 \% \text { OD }\left(100 \mathrm{~g} \mathrm{ha}^{-1}\right) \\
\text { (PoE) } f b \text { HW at } 40 \text { DAT }\end{array}$ & 100.8 & 394 & 14663 \\
\hline $\mathrm{T}_{7}$ - Pretilachlor $50 \%$ EC $0.75 \mathrm{~kg} \mathrm{ha}^{-1}(\mathrm{PE}) f b$ 2,4 D $1.0 \mathrm{~kg} \mathrm{ha}^{-1}(\mathrm{PoE})$ & 87.7 & 319 & 11835 \\
\hline $\mathrm{T}_{8}$ - Bispyribac sodium 10\% SC $25 \mathrm{~g} \mathrm{ha}^{-1}(\mathrm{PoE}) \mathrm{fb} \mathrm{HW}$ at 40 DAT & 88.5 & 326 & 12167 \\
\hline $\begin{array}{l}\mathrm{T}_{9} \text { - Flopyrauxifen- benzyl + penoxsulam } 12 \% \text { EC } 40.64 \mathrm{~g} \mathrm{ha}^{-1}(\mathrm{PoE}) \mathrm{fb} \mathrm{HW} \\
\text { at } 40 \text { DAT }\end{array}$ & 99.3 & 391 & 14448 \\
\hline $\begin{array}{l}\mathrm{T}_{10} \text { - Flopyrauxifen- benzyl + cyhalofop butyl } 10 \% \mathrm{EC} 150 \mathrm{~g} \mathrm{ha}^{-1} \text { (PoE) } f b \\
\text { HW at } 40 \text { DAT }\end{array}$ & 104.5 & 401 & 14953 \\
\hline $\mathrm{T}_{11}$ - Hand weeding at 20 and 40 DAT & 105.0 & 412 & 15014 \\
\hline$T_{12}$ - Unweeded control & 86.3 & 209 & 7732 \\
\hline $\mathbf{S E}(\mathbf{m}) \pm$ & 2.36 & 7.43 & 254.58 \\
\hline $\mathrm{CD}(\mathrm{P}=\mathbf{0 . 0 5})$ & 6.93 & 21.8 & 746.5 \\
\hline
\end{tabular}


Table.2 Effect of new herbicides and herbicide mixtures onyield attributes and yield of transplanted rice

\begin{tabular}{|c|c|c|c|c|c|}
\hline Treatments & 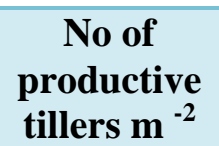 & $\begin{array}{l}\text { No. of filled } \\
\text { grains panicle }\end{array}$ & $\begin{array}{c}\text { Test } \\
\text { weight } \\
\text { (g) }\end{array}$ & $\begin{array}{l}\text { Grain yield } \\
\left(\mathrm{kg} \mathrm{ha}^{-1}\right)\end{array}$ & $\begin{array}{l}\text { Straw yield } \\
\left(\mathrm{kg} \mathrm{ha}^{-1}\right)\end{array}$ \\
\hline $\begin{array}{l}\mathrm{T}_{1} \text { - Penoxsulam } 0.97 \%\left(20 \mathrm{~g} \mathrm{ha}^{-1}\right)+\text { butachlor }(38.8 \%) \text { SE } 820 \\
\mathrm{~g} \mathrm{ha}^{-1}(\mathrm{PE}) f b \text { HW at } 30 \text { DAT }\end{array}$ & 325 & 140 & 12.90 & 5931 & 7004 \\
\hline $\begin{array}{l}\mathrm{T}_{2} \text { - Pyrazosulfuron-ethyl } 0.15 \%\left(15 \mathrm{~g} \mathrm{ha}^{-1}\right)+\text { pretilachlor } 6 \% \\
\text { GR }\left(600 \mathrm{~g} \mathrm{ha}^{-1}\right)(\text { PE) } f b \text { HW at } 30 \text { DAT }\end{array}$ & 334 & 144 & 13.00 & 6016 & 7045 \\
\hline $\begin{array}{l}\text { T }_{3} \text { - Orthosulfamuron + pretilachlor } 6 \%\left(600 \mathrm{~g} \mathrm{ha}^{-1}\right) \text { GR (PE) } \\
f b \text { HW at } 30 \text { DAT }\end{array}$ & 327 & 141 & 13.00 & 5977 & 7015 \\
\hline $\begin{array}{l}\mathrm{T}_{4} \text { - Ipfencarbazone } 25 \% \text { SC } 156.25 \mathrm{~g} \mathrm{ha}^{-1}(\mathrm{PE}) \mathrm{fb} \mathrm{HW} \text { at } 30 \\
\text { DAT }\end{array}$ & 315 & 130 & 12.87 & 5524 & 6471 \\
\hline $\mathrm{T}_{5}$ - Penoxsulam $2.65 \%$ OD $25 \mathrm{~g} \mathrm{ha}^{-1}(\mathrm{PoE}) \mathrm{fb} \mathrm{HW}$ at $40 \mathrm{DAT}$ & 313 & 127 & 12.80 & 5497 & 6425 \\
\hline $\begin{array}{l}\mathrm{T}_{6} \text { - Penoxsulam } 1.02 \%\left(20 \mathrm{~g} \mathrm{ha}^{-1}\right)+\text { cyhalofop butyl } 5.1 \% \text { OD } \\
\left(100 \mathrm{~g} \mathrm{ha}^{-1}\right)(\text { PoE }) f b \text { HW at } 40 \text { DAT }\end{array}$ & 351 & 157 & 13.07 & 6985 & 7818 \\
\hline $\begin{array}{l}\text { T }_{7} \text { - Pretilachlor } 50 \% \text { EC } 0.75 \mathrm{~kg} \mathrm{ha}^{-1}(\mathrm{PE}) f b 2,4 \text { D } 1.0 \mathrm{~kg} \mathrm{ha}^{-1} \\
\text { (PoE) }\end{array}$ & 307 & 122 & 12.09 & 5262 & 6187 \\
\hline $\begin{array}{l}\mathrm{T}_{8} \text { - Bispyribac sodium 10\% SC } 25 \mathrm{~g} \mathrm{ha}^{-1} \text { (PoE) } f b \mathrm{HW} \text { at } 40 \\
\text { DAT }\end{array}$ & 308 & 124 & 12.80 & 5333 & 6282 \\
\hline $\begin{array}{l}\text { T}_{9} \text { - Flopyrauxifen- benzyl + penoxsulam } 12 \% \text { EC } 40.64 \mathrm{~g} \mathrm{ha}^{-1} \\
\text { (PoE) } f b \text { HW at } 40 \text { DAT }\end{array}$ & 348 & 155 & 13.03 & 6867 & 7715 \\
\hline $\begin{array}{l}\mathrm{T}_{10} \text { - Flopyrauxifen- benzyl + cyhalofop butyl } 10 \% \text { EC } 150 \mathrm{~g} \\
\text { ha }^{-1} \text { (PoE) } f b \text { HW at } 40 \text { DAT }\end{array}$ & 355 & 161 & 13.20 & 7045 & 7921 \\
\hline $\mathrm{T}_{11}$ - Hand weeding at 20 and $40 \mathrm{DAT}$ & 356 & 162 & 13.23 & 7120 & 7992 \\
\hline$T_{12}$ - Unweeded control & 225 & 118 & 12.40 & 3110 & 4219 \\
\hline $\mathrm{SE}(\mathrm{m}) \pm$ & 6.04 & 3.73 & 11.00 & 146.3 & 171.54 \\
\hline CD $(P=0.05)$ & 17.73 & 10.95 & NS & 429.0 & 503.02 \\
\hline
\end{tabular}


Table.3 Effect of new herbicides and herbicide mixtures on weed density and dry weight at 30 DAT

\begin{tabular}{|c|c|c|}
\hline Treatments & $\begin{array}{l}\text { Weed density } \\
\left(\text { No. } \mathrm{m}^{-2}\right)\end{array}$ & $\begin{array}{c}\text { Weed dry } \\
\text { weight }\left(\mathrm{g} \mathrm{m}^{-2}\right)\end{array}$ \\
\hline $\begin{array}{l}\text { T }_{1} \text { - Penoxsulam } 0.97 \%\left(20 \mathrm{~g} \mathrm{ha}^{-1}\right)+\text { butachlor }(38.8 \%) \text { SE } 820 \mathrm{~g} \mathrm{ha}^{-1} \\
\text { (PE) } f b \text { HW at } 30 \text { DAT }\end{array}$ & $4.5(19.0)$ & $3.9(14.5)$ \\
\hline $\begin{array}{l}\mathrm{T}_{2} \text { - Pyrazosulfuron-ethyl } 0.15 \%\left(15 \mathrm{~g} \mathrm{ha}^{-1}\right)+\text { pretilachlor } 6 \% \text { GR }(600 \mathrm{~g} \\
\left.\text { ha }^{-1}\right) \text { (PE) } f b \text { HW at } 30 \text { DAT }\end{array}$ & 4.1(15.7) & $3.8(13.3)$ \\
\hline $\begin{array}{l}\text { T }_{3} \text { - Orthosulfamuron + pretilachlor } 6 \%\left(600 \mathrm{~g} \mathrm{ha}^{-1}\right) \mathrm{GR}(\mathrm{PE}) \mathrm{fb} \mathrm{HW} \text { at } \\
30 \text { DAT }\end{array}$ & $4.4(18.3)$ & $3.9(14.0)$ \\
\hline $\mathrm{T}_{4}$ - Ipfencarbazone $25 \% \mathrm{SC} 156.25 \mathrm{~g} \mathrm{ha}^{-1}(\mathrm{PE}) \mathrm{fb} \mathrm{HW}$ at $30 \mathrm{DAT}$ & $4.7(21.0)$ & $4.2(16.8)$ \\
\hline $\mathrm{T}_{5}$ - Penoxsulam $2.65 \%$ OD $25 \mathrm{~g} \mathrm{ha}^{-1}$ (PoE) $f b \mathrm{HW}$ at $40 \mathrm{DAT}$ & $5.0(23.7)$ & $4.3(18.0)$ \\
\hline $\begin{array}{l}\mathrm{T}_{6} \text { - Penoxsulam } 1.02 \%\left(20 \mathrm{~g} \mathrm{ha}^{-1}\right)+\text { cyhalofop butyl } 5.1 \% \text { OD }\left(100 \mathrm{~g} \mathrm{ha}^{-}\right. \\
\left.1^{-}\right)(\text {PoE }) \mathrm{fb} \text { HW at } 40 \text { DAT }\end{array}$ & $3.3(9.7)$ & $2.9(7.4)$ \\
\hline $\mathrm{T}_{7}$ - Pretilachlor $50 \%$ EC $0.75 \mathrm{~kg} \mathrm{ha}^{-1}$ (PE) fb 2,4 D $1.0 \mathrm{~kg} \mathrm{ha}^{-1}(\mathrm{PoE})$ & $5.1(25.0)$ & $4.5(19.2)$ \\
\hline $\mathrm{T}_{8}$ - Bispyribac sodium 10\% SC $25 \mathrm{~g} \mathrm{ha}^{-1}$ (PoE) $f b$ HW at 40 DAT & $5.0(24.3)$ & $4.4(18.6)$ \\
\hline $\begin{array}{l}\text { T }_{9} \text { - Flopyrauxifen- benzyl + penoxsulam } 12 \% \text { EC } 40.64 \mathrm{~g} \mathrm{ha}^{-1} \text { (PoE) } f b \\
\text { HW at } 40 \text { DAT }\end{array}$ & $3.3(10.0)$ & $2.9(7.7)$ \\
\hline $\begin{array}{l}\mathrm{T}_{10} \text { - Flopyrauxifen- benzyl + cyhalofop butyl } 10 \% \text { EC } 150 \mathrm{~g} \mathrm{ha}^{-1} \text { (PoE) } \\
f b \text { HW at } 40 \text { DAT }\end{array}$ & $3.1(8.7)$ & $2.7(6.3)$ \\
\hline $\mathrm{T}_{11}$ - Hand weeding at 20 and $40 \mathrm{DAT}$ & $2.8(7.3)$ & $2.6(6.0)$ \\
\hline $\mathbf{T}_{12}$ - Unweeded control & $8.7(74.0)$ & $8.2(65.7)$ \\
\hline $\mathrm{SE}(\mathbf{m}) \pm$ & 0.18 & 0.17 \\
\hline $\mathrm{CD}(\mathrm{P}=0.05)$ & 0.53 & 0.51 \\
\hline
\end{tabular}

** Values in the parenthesis are original and $(\sqrt{\mathrm{x}}+1)$ transformed 


\section{Yield attributes and yield}

Yield attributes and yield significantly influenced by different weed management practices. Higher number of productive tillers $\mathrm{m}^{-2}$, number of filled grains panicle ${ }^{-1}$, grain and straw yield were registered with hand weeding at 20 and 40 DAT which was statistically on par with the flopyrauxifenbenzyl + cyhalofop butyl 10\% EC $150 \mathrm{~g} \mathrm{ha}^{-1}$ (PoE) $f b$ hand weeding at 40 DAT, penoxsulam $1.02 \%\left(20 \mathrm{~g} \mathrm{ha}^{-1}\right)+$ cyhalofop butyl 5.1\% OD (100 $\mathrm{g} \mathrm{ha}^{-1}$ ) (PoE) $f b$ hand weeding at 40 DAT and flopyrauxifen- benzyl + penoxsulam 12\% EC $40.64 \mathrm{~g} \mathrm{ha}^{-1}$ (PoE) $f b$ hand weeding at $40 \mathrm{DAT}$. These treatments followed by pyrazosulfuron-ethyl $0.15 \%$ @ $15 \mathrm{~g} \mathrm{ha}^{-1}+$ pretilachlor $6 \%$ GR @ $600 \mathrm{~g} \mathrm{ha}^{-1}$ (PE) $f b$ hand weeding at 30 DAT, orthosulfamuron + pretilachlor $6 \%$ @ $600 \mathrm{~g}$ ha ${ }^{-1} \mathrm{GR}$ (PE) $f b$ hand weeding at 30 DAT and penoxsulam $0.97 \%$ @ $20 \mathrm{~g} \mathrm{ha}^{-1}+$ butachlor $38.8 \%$ SE @ $820 \mathrm{~g} \mathrm{ha}^{-1}$ (PE) $f b$ hand weeding at 30 DAT and which were superior over remaining treatments. Then followed by ipfencarbazone 25\% SC @ $156.25 \mathrm{~g} \mathrm{ha}^{-1}$ (PE) $f b$ hand weeding at 30 DAT, penoxsulam $2.65 \%$ OD @ $25 \mathrm{~g} \mathrm{ha}^{-1}$ (PoE) $f b$ hand weeding at 40 DAT, bispyribac-sodium $10 \%$ SC $25 \mathrm{~g} \mathrm{ha}^{-1}$ (PoE) $f b$ hand weeding at 40 DAT and pretilachlor (PE) $50 \%$ EC @ 0.75 $\mathrm{kg}$ ha ${ }^{-1} f b 2,4-\mathrm{D} 1.0 \mathrm{~kg} \mathrm{ha}^{-1}$ (PoE). Un weeded control plot recorded significantly lowernumber of yield attributes and yield. Weed management practices not only reduce weed density and dry matter allows the plant to use available resources which resulted in higher growth parameters and yield attributes ultimately led to higher yield over un weeded control. Similar reports were by Chowdhary and Dixit (2018), Singh et al., (2019) and Ramesha et al., (2019).

\section{Weed density and weed dry weight}

Lower total weed density and total weed dry weight at 30 DAT, was observed with hand weeding at 20 and 40 DAT and which was statistically comparable to the flopyrauxifenbenzyl + cyhalofop-butyl 10\% EC $150 \mathrm{~g}$ ha ${ }^{1}(\mathrm{PoE}) f b$ hand weeding at 40 DAT, penoxsulam 1.02\%@20 $\mathrm{g} \mathrm{ha}^{-1}+$ cyhalofop butyl 5.1\% OD @ $100 \mathrm{~g} \mathrm{ha}^{-1}$ (PoE) $f b$ hand weeding at 40 DAT and flopyrauxifen-benzyl + penoxsulam $12 \%$ EC @ $40.64 \mathrm{~g} \mathrm{ha}^{-1}$ $(\mathrm{PoE}) f b$ hand weeding at 40 DAT. These treatments followed pyrazosulfuron-ethyl $0.15 \% @ 15 \mathrm{~g} \mathrm{ha}^{-1}+$ pretilachlor 6\% GR @ $600 \mathrm{~g} \mathrm{ha}^{-1}(\mathrm{PE}) \mathrm{fb}$ hand weeding at $30 \mathrm{DAT}$, orthosulfamuron + pretilachlor $6 \%$ @ $600 \mathrm{~g}$ ha ${ }^{-1}$ GR (PE) $f b$ hand weeding at 30 DAT and penoxsulam $0.97 \%$ @ $20 \mathrm{~g} \mathrm{ha}^{-1}+$ butachlor $38.8 \%$ SE @ $820 \mathrm{~g} \mathrm{ha}^{-1}(\mathrm{PE}) f b$ hand weeding at 30 DAT were recorded lower total weed density and weed dry weight than rest of treatments. Then followed by ipfencarbazone $25 \%$ SC @ $156.25 \mathrm{~g} \mathrm{ha}{ }^{-1}(\mathrm{PE}) f b$ hand weeding at 30 DAT, penoxsulam $2.65 \%$ OD @ $25 \mathrm{~g} \mathrm{ha}^{-1}$ (PoE) $f b$ hand weeding at 40 DAT, bispyribac-sodium 10\% SC $25 \mathrm{~g}$ ha ${ }^{1}(\mathrm{PoE}) f b$ hand weeding at 40 DAT and pretilachlor (PE) $50 \%$ EC @ $0.75 \mathrm{~kg} \mathrm{ha}^{-1} f b$ 2,4-D $1.0 \mathrm{~kg} \mathrm{ha}^{-1}(\mathrm{PoE})$ were on par with each other. Higher weed density and weed dry weight was recorded with un weeded control over all the treatments. Lower weed density in different weed management practices might be due to effective control of weeds Hossain and Mondal (2014) and Yadav et al., (2019b).

In conclusion the hand weeding twice 20 and 40 DAT, Post emergence (2- 3 leaf stage of weeds) application of herbicide mixtures flopyrauxifen- benzyl + cyhalofop butyl 10\% EC $150 \mathrm{~g} \mathrm{ha}^{-1}$, Penoxsulam 1.02\% (20 $\left.\mathrm{g} \mathrm{ha}^{-1}\right)$ + cyhalofop butyl 5.1\% OD (100 $\left.\mathrm{g} \mathrm{ha}^{-1}\right)$ and flopyrauxifen- benzyl + penoxsulam $12 \%$ EC $40.64 \mathrm{~g} \mathrm{ha}^{-1}$ along with hand weeding at 40 DAT were effective in influencing of growth parameters, yield attributes and yield of transplanted rice. 


\section{References}

Choudhary, V.K and Dixit, A. 2018. Herbicide weed management on weed dynamics, crop growth and yield in direct-seeded rice. Indian Journal of Weed Science. 50(1): 6-12.

Duary, B., Teja, K. C and Soren, U. 2015. Management of composite weed flora of transplanted rice by herbicides. Indian Journal of Weed Science. 47(4): 349-352.

Mohapatra, S., Tripathy, S.K., Nayak, B.R and Mohanty, A.K. 2017. Efficacy of pre-emergence herbicides for control of complex weed flora in transplanted rice. Indian Journal of Weed Science. 49 (3): 216-218.

Ramesha, Y. M., Anand, S. R., Krishnamurthy, D and Bhanuvally, M. 2019. Weed management effect to increase grain yield in dry directseeded rice. Indian Journal of Weed Science. 51(1): 6-9.

Rana, A., Rana, M. C., Rana, S. S., Sharma, N and Kumar, S. 2018. Weed control by pyrazosulfuron-ethyl and its influence on yield and economics of transplanted rice. Indian Journal of Weed Science. 50(4): 309-314.

Singh, K., Singh, S and Pannu, R. K. 2019. Efficacy of pendimethalin and cyhalofop-butyl+ penoxsulam against major grass weeds of direct-seeded rice. Indian Journal of Weed Science. 51 (3): 227-231.

Yadav, D. B., Singh, N., Duhan, A., Yadav, A and Punia, S. S. 2019. Penoxsulam influence on weed complex and productivity of transplanted rice and its residual effects in rice-wheat cropping system. Indian Journal of Weed Science. 51(1): 10-14.

Yakadri, M., Madhavi, M., Ramprakash, T and Rani, L. 2016. Herbicide combinations for control of complex weed flora in transplanted rice. Indian Journal of Weed Science. 48 (2): 155157.

\section{How to cite this article:}

Venkatesh, B., Y. S. Parameswari, M. Madhavi and Ram Prakash, T. 2020. Effect of New Herbicides and Herbicide Mixtures on Growth and Yield of Transplanted Rice. Int.J.Curr.Microbiol.App.Sci. 9(07): 2201-2207. doi: https://doi.org/10.20546/ijcmas.2020.907.257 